TRANSACTIONS OF THE

AMERICAN MATHEMATICAL SOCIETY

Volume 359, Number 5, May 2007, Pages 2407-2420

S 0002-9947(06)04163-8

Article electronically published on December 5, 2006

\title{
THIN STATIONARY SETS AND DISJOINT CLUB SEQUENCES
}

\author{
SY-DAVID FRIEDMAN AND JOHN KRUEGER
}

\begin{abstract}
We describe two opposing combinatorial properties related to adding clubs to $\omega_{2}$ : the existence of a thin stationary subset of $P_{\omega_{1}}\left(\omega_{2}\right)$ and the existence of a disjoint club sequence on $\omega_{2}$. A special Aronszajn tree on $\omega_{2}$ implies there exists a thin stationary set. If there exists a disjoint club sequence, then there is no thin stationary set, and moreover there is a fat stationary subset of $\omega_{2}$ which cannot acquire a club subset by any forcing poset which preserves $\omega_{1}$ and $\omega_{2}$. We prove that the existence of a disjoint club sequence follows from Martin's Maximum and is equiconsistent with a Mahlo cardinal.
\end{abstract}

Suppose that $S$ is a fat stationary subset of $\omega_{2}$, that is, for every club set $C \subseteq$ $\omega_{2}, S \cap C$ contains a closed subset with order type $\omega_{1}+1$. A number of forcing posets have been defined which add a club subset to $S$ and preserve cardinals under various assumptions. Abraham and Shelah 1 proved that, assuming $\mathrm{CH}$, the poset consisting of closed bounded subsets of $S$ ordered by end-extension adds a club subset to $S$ and is $\omega_{1}$-distributive. S. Friedman [5] discovered a different poset for adding a club subset to a fat set $S \subseteq \omega_{2}$ with finite conditions 1 This finite club poset preserves all cardinals provided that there exists a thin stationary subset of $P_{\omega_{1}}\left(\omega_{2}\right)$, that is, a stationary set $T \subseteq P_{\omega_{1}}\left(\omega_{2}\right)$ such that for all $\beta<\omega_{2}$, $|\{a \cap \beta: a \in T\}| \leq \omega_{1}$. This notion of stationarity appears in [9] and was discovered independently by Friedman. The question remained whether it is always possible to add a club subset to a given fat set and preserve cardinals, without any assumptions.

J. Krueger introduced a combinatorial principle on $\omega_{2}$ which asserts the existence of a disjoint club sequence, which is a pairwise disjoint sequence $\left\langle\mathcal{C}_{\alpha}: \alpha \in A\right\rangle$ indexed by a stationary subset of $\omega_{2} \cap \operatorname{cof}\left(\omega_{1}\right)$, where each $\mathcal{C}_{\alpha}$ is club in $P_{\omega_{1}}(\alpha)$. Krueger proved that the existence of such a sequence implies there is a fat stationary set $S \subseteq \omega_{2}$ which cannot acquire a club subset by any forcing poset which preserves $\omega_{1}$ and $\omega_{2}$.

We prove that a special Aronszajn tree on $\omega_{2}$ implies there exists a thin stationary subset of $P_{\omega_{1}}\left(\omega_{2}\right)$. On the other hand assuming Martin's Maximum there exists a disjoint club sequence on $\omega_{2}$. Moreover, we have the following equiconsistency result.

Theorem 0.1. Each of the following statements is equiconsistent with a Mahlo cardinal: (1) There does not exist a thin stationary subset of $P_{\omega_{1}}\left(\omega_{2}\right)$. (2) There exists a disjoint club sequence on $\omega_{2}$. (3) There exists a fat stationary set $S \subseteq \omega_{2}$

Received by the editors June 28, 2005.

2000 Mathematics Subject Classification. Primary 03E35, 03E40.

The authors were supported by FWF project number P16790-N04.

${ }^{1}$ A similar poset was defined independently by Mitchell [7]. 
such that any forcing poset which preserves $\omega_{1}$ and $\omega_{2}$ does not add a club subset to $S$.

Our proof of this theorem gives a totally different construction of the following result of Mitchell [8]: If $\kappa$ is Mahlo in $L$, then there is a generic extension of $L$ in which $\kappa=\omega_{2}$ and there is no special Aronszajn tree on $\omega_{2}$. The consistency of Theorem 0.1(3) provides a negative solution to the following problem of Abraham and Shelah [1]: If $S \subseteq \omega_{2}$ is fat, does there exist an $\omega_{1}$-distributive forcing poset which adds a club subset to $S$ ?

Section 1 outlines notation and background material. In Section 2 we discuss thin stationarity and prove that a special Aronszajn tree implies the existence of a thin stationary set. In Section 3 we introduce disjoint club sequences and prove that the existence of such a sequence implies there is a fat stationary set in $\omega_{2}$ which cannot acquire a club subset by any forcing poset which preserves $\omega_{1}$ and $\omega_{2}$. In Section 4 we prove that Martin's Maximum implies there exists a disjoint club sequence. In Section 5 we construct a model in which there is a disjoint club sequence using an RCS iteration up to a Mahlo cardinal.

Sections 3 and 4 are due for the most part to J. Krueger. We would like to thank Boban Veličković and Mirna Džamonja for pointing out Theorem 2.3 to the authors.

\section{Preliminaries}

For a set $X$ which contains $\omega_{1}, P_{\omega_{1}}(X)$ denotes the collection of countable subsets of $X$. A set $C \subseteq P_{\omega_{1}}(X)$ is club if it is closed under unions of countable increasing sequences and is cofinal. A set $S \subseteq P_{\omega_{1}}(X)$ is stationary if it meets every club. If $C \subseteq P_{\omega_{1}}(X)$ is club, then there exists a function $F: X^{<\omega} \rightarrow X$ such that every $a$ in $P_{\omega_{1}}(X)$ closed under $F$ is in $C$. If $F: X^{<\omega} \rightarrow P_{\omega_{1}}(X)$ is a function and $Y \subseteq X$, we say that $Y$ is closed under $F$ if for all $\vec{\gamma}$ from $Y^{<\omega}, F(\vec{\gamma}) \subseteq Y$. A partial function $H: P_{\omega_{1}}(X) \rightarrow X$ is regressive if for all $a$ in the domain of $H, H(a)$ is a member of a. Fodor's Lemma asserts that whenever $S \subseteq P_{\omega_{1}}(X)$ is stationary and $H: S \rightarrow X$ is a total regressive function, there is a stationary set $S^{*} \subseteq S$ and a set $x$ in $X$ such that for all $a$ in $S^{*}, H(a)=x$.

If $\kappa$ is a regular cardinal let $\operatorname{cof}(\kappa)$ (respectively, $\operatorname{cof}(<\kappa)$ ) denote the class of ordinals with cofinality $\kappa$ (respectively, cofinality less than $\kappa$ ). If $A$ is a cofinal subset of a cardinal $\lambda$ and $\kappa<\lambda$, we write for example $A \cup \operatorname{cof}(\kappa)$ to abbreviate $A \cup(\lambda \cap \operatorname{cof}(\kappa))$.

A stationary set $S \subseteq \kappa$ is fat if for every club $C \subseteq \kappa, S \cap C$ contains closed subsets with arbitrarily large order types less than $\kappa$. If $\kappa$ is the successor of a regular uncountable cardinal $\mu$, this is equivalent to the statement that for every club $C \subseteq \kappa, S \cap C$ contains a closed subset with order type $\mu+1$. In particular, if $A \subseteq \kappa^{+} \cap \operatorname{cof}(\mu)$ is stationary, then $A \cup \operatorname{cof}(<\mu)$ is fat.

We write $\theta \gg \kappa$ to indicate $\theta$ is larger than $2^{2^{|H(\kappa)|}}$.

A tree $\mathcal{T}$ is a special Aronszajn tree on $\omega_{2}$ if:

(1) $\mathcal{T}$ has height $\omega_{2}$ and each level has size less than $\omega_{2}$,

(2) each node in $\mathcal{T}$ is an injective function $f: \alpha \rightarrow \omega_{1}$ for some $\alpha<\omega_{2}$,

(3) the ordering on $\mathcal{T}$ is by extension of functions, and if $f$ is in $\mathcal{T}$, then $f \uparrow \beta$ is in $\mathcal{T}$ for all $\beta<\operatorname{dom}(f)$.

By $[8]$ if there does not exist a special Aronszajn tree on $\omega_{2}$, then $\omega_{2}$ is a Mahlo cardinal in $L$. 
If $V$ is a transitive model of ZFC, we say that $W$ is an outer model of $V$ if $W$ is a transitive model of ZFC such that $V \subseteq W$ and $W$ has the same ordinals as $V$.

A forcing poset $\mathbb{P}$ is $\kappa$-distributive if forcing with $\mathbb{P}$ does not add any new sets of ordinals with size $\kappa$.

If $\mathbb{P}$ is a forcing poset, $\dot{a}$ is a $\mathbb{P}$-name, and $G$ is a generic filter for $\mathbb{P}$, we write $a$ for the set $\dot{a}^{G}$.

Martin's Maximum is the statement that whenever $\mathbb{P}$ is a forcing poset which preserves stationary subsets of $\omega_{1}$, then for any collection $\mathcal{D}$ of dense subsets of $\mathbb{P}$ with $|\mathcal{D}| \leq \omega_{1}$, there is a filter $G \subseteq \mathbb{P}$ which intersects each dense set in $\mathcal{D}$.

A forcing poset $\mathbb{P}$ is proper if for all sufficiently large regular cardinals $\theta>2^{|\mathbb{P}|}$, there is a club of countable elementary substructures $N$ of $\langle H(\theta), \in\rangle$ such that for all $p$ in $N \cap \mathbb{P}$, there is $q \leq p$ which is generic for $N$, i.e. $q$ forces $N[\dot{G}] \cap \mathbf{O n}=N \cap \mathbf{O n}$. If $\mathbb{P}$ is proper, then $\mathbb{P}$ preserves $\omega_{1}$ and preserves stationary subsets of $P_{\omega_{1}}(\lambda)$ for all $\lambda \geq \omega_{1}$. A forcing poset $\mathbb{P}$ is semiproper if the same statement holds as above except the requirement that $q$ is generic is replaced by $q$ being semigeneric, i.e. $q$ forces $N[\dot{G}] \cap \omega_{1}=N \cap \omega_{1}$. If $\mathbb{P}$ is semigeneric, then $\mathbb{P}$ preserves $\omega_{1}$ and preserves stationary subsets of $\omega_{1}$.

If $\mathbb{P}$ is $\omega_{1}$-c.c. and $N$ is a countable elementary substructure of $H(\theta)$, then $\mathbb{P}$ forces $N[\dot{G}] \cap \mathbf{O n}=N \cap \mathbf{O n}$; so every condition in $\mathbb{P}$ is generic for $N$.

We let ${ }^{<\omega}$ On denote the class of finite strictly increasing sequences of ordinals. If $\eta$ and $\nu$ are in ${ }^{<\omega} \mathbf{O n}$, write $\eta \unlhd \nu$ if $\eta$ is an initial segment of $\nu$, and write $\eta \triangleleft \nu$ if $\eta \unlhd \nu$ and $\eta \neq \nu$. Let $l(\eta)$ denote the length of $\eta$. A set $T \subseteq{ }^{<\omega} \mathbf{O n}$ is a tree if for all $\eta$ in $T$ and $k<l(\eta), \eta \uparrow k$ is in $T$. A cofinal branch of $T$ is a function $b: \omega \rightarrow \kappa$ such that for all $n<\omega, b\lceil n$ is in $T$.

Suppose $I$ is an ideal on a set $X$. Then $I^{+}$is the collection of subsets of $X$ which are not in $I$. If $S$ is in $I^{+}$let $I \uparrow S$ denote the ideal $I \cap \mathcal{P}(S)$. For example if $I=N S_{\kappa}$, the ideal of non-stationary subsets of $\kappa$, a set $S$ is in $I^{+}$iff $S$ is stationary. In this case $N S_{\kappa}\left\lceil S\right.$ is the ideal of non-stationary subsets of $S$, and $\left(N S_{\kappa}\lceil S)^{+}\right.$is the collection of stationary subsets of $S$.

If $\kappa$ is regular and $\lambda \geq \kappa$ is a cardinal, then $\operatorname{CoLL}(\kappa, \lambda)$ is a forcing poset for collapsing $\lambda$ to have cardinality $\kappa$ : Conditions are partial functions $p: \kappa \rightarrow \lambda$ with size less than $\kappa$, ordered by an extension of functions.

\section{THIN STATIONARY SETS}

Let $T$ be a cofinal subset of $P_{\omega_{1}}\left(\omega_{2}\right)$. We say that $T$ is thin if for all $\beta<\omega_{2}$ the set $\{a \cap \beta: a \in T\}$ has size less than $\omega_{2}$. Note that if $\mathrm{CH}$ holds, then $P_{\omega_{1}}\left(\omega_{2}\right)$ itself is thin. A set $S \subseteq P_{\omega_{1}}\left(\omega_{2}\right)$ is closed under initial segments if for all $a$ in $S$ and $\beta<\omega_{2}, a \cap \beta$ is in $S$.

Lemma 2.1. If $S \subseteq P_{\omega_{1}}\left(\omega_{2}\right)$ is stationary and closed under initial segments, then for all uncountable $\beta<\omega_{2}$, the set $S \cap P_{\omega_{1}}(\beta)$ is stationary in $P_{\omega_{1}}(\beta)$.

Proof. Consider $\beta<\omega_{2}$ and let $C \subseteq P_{\omega_{1}}(\beta)$ be a club set. Then the set $D=$ $\left\{a \in P_{\omega_{1}}\left(\omega_{2}\right): a \cap \beta \in C\right\}$ is a club subset of $P_{\omega_{1}}\left(\omega_{2}\right)$. Fix $a$ in $S \cap D$. Since $S$ is closed under initial segments, $a \cap \beta$ is in $S \cap C$.

Lemma 2.2. If there exists a thin stationary subset of $P_{\omega_{1}}\left(\omega_{2}\right)$, then there is a thin stationary set $S$ such that for all uncountable $\beta<\omega_{2}, S \cap P_{\omega_{1}}(\beta)$ is stationary in $P_{\omega_{1}}(\beta)$. 
Proof. Let $T$ be a thin stationary set. Define $S=\left\{a \cap \beta: a \in T, \beta<\omega_{2}\right\}$. Then $S$ is thin stationary and closed under initial segments.

A set $S \subseteq P_{\omega_{1}}\left(\omega_{2}\right)$ is a local club if there is a club set $C \subseteq \omega_{2}$ such that for all uncountable $\alpha$ in $C, S \cap P_{\omega_{1}}(\alpha)$ contains a club in $P_{\omega_{1}}(\alpha)$ (see [3]). Note that local clubs are stationary.

Theorem 2.3. If there is a special Aronszajn tree on $\omega_{2}$, then there is a thin local club subset of $P_{\omega_{1}}\left(\omega_{2}\right)$.

Proof. Let $\mathcal{T}$ be a special Aronszajn tree on $\omega_{2}$. For each $f$ in $\mathcal{T}$ with $\operatorname{dom}(f) \geq \omega_{1}$, define $S_{f}=\left\{\{\alpha \in \operatorname{dom}(f): f(\alpha)<i\}: i<\omega_{1}\right\}$. Note that $S_{f}$ is a club subset of $P_{\omega_{1}}(\operatorname{dom} f)$. For each uncountable $\beta<\omega_{2}$ define $S_{\beta}=\bigcup\left\{S_{f}: f \in \mathcal{T}, \operatorname{dom}(f)=\beta\right\}$. Then $S_{\beta}$ has size $\omega_{1}$. Now define $S=\bigcup\left\{S_{\beta}: \omega_{1} \leq \beta<\omega_{2}\right\}$. Clearly $S$ is a local club. To show $S$ is thin, it suffices to prove that whenever $\beta<\gamma$ are uncountable and $a$ is in $S_{\gamma}$, then $a \cap \beta$ is in $S_{\beta}$. Fix $f$ in $\mathcal{T}$ and $i<\omega_{1}$ such that $a=f^{-1}$ " $i$. Then $f\left\lceil\beta\right.$ is in $\mathcal{T}$, so $\left(f\lceil\beta)^{-1} " i\right.$ is in $\mathcal{S}_{\beta}$. But $\left(f\lceil\beta)^{-1} " i=\left(f^{-1} " i\right) \cap \beta=a \cap \beta\right.$.

In later sections of the paper we will construct models in which there does not exist a thin stationary subset of $P_{\omega_{1}}\left(\omega_{2}\right)$. Theorem 2.3 shows that in such a model there cannot exist a special Aronszajn tree on $\omega_{2}$, so by $8 \omega_{2}$ is Mahlo in $L$. Mitchell 8] constructed a model in which there is no special Aronszajn tree on $\omega_{2}$ by collapsing a Mahlo cardinal in $L$ to become $\omega_{2}$ with a proper forcing poset. However, in Mitchell's model the set $\left(P_{\omega_{1}}(\kappa)\right)^{L}$ is a thin stationary subset of $P_{\omega_{1}}\left(\omega_{2}\right)$.

Lemma 2.4. Suppose $S \subseteq P_{\omega_{1}}\left(\omega_{2}\right)$ is a local club. Then $S$ is a local club in any outer model $W$ with the same $\omega_{1}$ and $\omega_{2}$.

Proof. Let $C$ be a club subset of $\omega_{2}$ such that for every uncountable $\alpha$ in $C, S \cap$ $P_{\omega_{1}}(\alpha)$ contains a club in $P_{\omega_{1}}(\alpha)$. Then $C$ remains club in $W$. For each uncountable $\alpha$ in $C$, fix a bijection $g_{\alpha}: \omega_{1} \rightarrow \alpha$. Then $\left\{g_{\alpha} " i: i<\omega_{1}\right\}$ is a club subset of $P_{\omega_{1}}(\alpha)$. By intersecting this club with $S$, we get a club subset of $S \cap P_{\omega_{1}}(\alpha)$ of the form $\left\{a_{i}^{\alpha}: i<\omega_{1}\right\}$ which is increasing and continuous. Clearly this set remains a club subset of $P_{\omega_{1}}(\alpha)$ in $W$.

Proposition 2.5. (1) Suppose there exists a thin local club in $P_{\omega_{1}}\left(\omega_{2}\right)$. Then there exists a thin local club in any outer model with the same $\omega_{1}$ and $\omega_{2}$. (2) Suppose $\kappa$ is a cardinal such that for all $\mu<\kappa, \mu^{\omega}<\kappa$, and assume $\mathbb{P}$ is a proper forcing poset which collapses $\kappa$ to become $\omega_{2}$. Then $\mathbb{P}$ forces that there is a thin stationary subset of $P_{\omega_{1}}\left(\omega_{2}\right)$.

Proof. (1) is immediate from Lemma 2.4 and the absoluteness of thinness. (2) Let $G$ be generic for $\mathbb{P}$ over $V$ and work in $V[G]$. Since $\mathbb{P}$ is proper, $\omega_{1}$ is preserved and the set $S=\left(P_{\omega_{1}}(\kappa)\right)^{V}$ is stationary in $P_{\omega_{1}}\left(\omega_{2}\right)$. We claim that $S$ is thin. If $\beta<\omega_{2}$, then $\{a \cap \beta: a \in S\}=\left(P_{\omega_{1}}(\beta)\right)^{V}$, and $\left|\left(P_{\omega_{1}}(\beta)\right)^{V}\right| \leq\left|\left(P_{\omega_{1}}(\beta)\right)^{V}\right|^{V}=\left|\left(\beta^{\omega}\right)^{V}\right|^{V}<\kappa$.

As we mentioned above, if $\mathrm{CH}$ holds, then the set $P_{\omega_{1}}\left(\omega_{2}\right)$ itself is thin. We show on the other hand that if $\mathrm{CH}$ fails, then no club subset of $P_{\omega_{1}}\left(\omega_{2}\right)$ is thin. The proof is due to Baumgartner and Taylor 2] who showed that for any club set $C \subseteq P_{\omega_{1}}\left(\omega_{2}\right)$, there is a countable set $A \subseteq \omega_{2}$ such that $C \cap \mathcal{P}(A)$ has size at least $2^{\omega}$. Their method of proof is described in the next lemma; we include the proof since we will use similar arguments later in the paper. 
Lemma 2.6. Suppose $Z$ is a stationary subset of $\omega_{2} \cap \operatorname{cof}(\omega)$ and for each $\alpha$ in $Z$, $M_{\alpha}$ is a countable cofinal subset of $\alpha$. Then there is a sequence $\left\langle Z_{s}, \xi_{s}: s \in{ }^{<\omega} 2\right\rangle$ satisfying:

(1) each $Z_{s}$ is a stationary subset of $Z$,

(2) if $s \unlhd t$, then $Z_{t} \subseteq Z_{s}$,

(3) if $\alpha$ is in $Z_{s}$, then $\xi_{s}$ is in $M_{\alpha}$,

(4) if $\alpha$ is in $Z_{s \wedge 0}$ and $\beta$ is in $Z_{s \wedge 1}$, then $\xi_{s \wedge 0}$ is not in $M_{\beta}$ and $\xi_{s \wedge 1}$ is not in $M_{\alpha}$.

Proof. Let $Z_{\langle\rangle}=Z$ and let $\xi_{\langle\rangle}=0$. Suppose $Z_{s}$ is given. Define $X_{s}$ as the set of $\xi$ in $\omega_{2}$ such that the set $\left\{\alpha \in Z_{s}: \xi \in M_{\alpha}\right\}$ is stationary. A straightforward argument using Fodor's Lemma shows that $X_{s}$ is unbounded in $\omega_{2}$. For each $\alpha$ in $Z_{s}$ such that $X_{s} \cap \alpha$ has size $\omega_{1}$, there exists $\xi<\alpha$ in $X_{s}$ such that $\xi$ is not in $M_{\alpha}$. By Fodor's Lemma there is a stationary set $Z_{s \wedge 1}^{\prime} \subseteq Z_{s}$ and $\xi_{s \wedge 0}$ in $X_{s}$ such that for all $\alpha$ in $Z_{s^{\wedge} 1}^{\prime}, \xi_{s^{\wedge} 0}$ is not in $M_{\alpha}$. Let $Z_{s \wedge 0}^{\prime}$ denote the set of $\alpha$ in $Z_{s}$ such that $\xi_{s \wedge 0}$ is in $M_{\alpha}$, which is stationary since $\xi_{s \wedge 0}$ is in $X_{s}$. Now define $Y_{s}$ as the set of $\xi$ in $\omega_{2}$ such that $\left\{\alpha \in Z_{s^{\wedge} 1}^{\prime}: \xi \in M_{\alpha}\right\}$ is stationary. Then $Y_{s}$ is unbounded in $\omega_{2}$. So for each $\alpha$ in $Z_{s^{\wedge} 0}^{\prime}$ such that $Y_{s} \cap \alpha$ has size $\omega_{1}$, there is $\xi<\alpha$ in $Y_{s}$ which is not in $M_{\alpha}$. By Fodor's Lemma there is $\xi_{s^{\wedge} 1}$ in $Y_{s}$ and $Z_{s^{\wedge} 0} \subseteq Z_{s \wedge 0}^{\prime}$ stationary such that for all $\alpha$ in $Z_{s^{\wedge} 0}, \xi_{s^{\wedge} 1}$ is not in $M_{\alpha}$. Now define $Z_{s^{\wedge} 1}$ as the set of $\alpha$ in $Z_{s \wedge 1}^{\prime}$ such that $\xi_{s^{\wedge} 1}$ is in $M_{\alpha}$.

Theorem 2.7 (Baumgartner and Taylor). If $C \subseteq P_{\omega_{1}}\left(\omega_{2}\right)$ is club, then there is a countable set $A \subseteq \omega_{2}$ such that $C \cap \mathcal{P}(A)$ has size at least $2^{\omega}$. Hence if $\mathrm{CH}$ fails, then there does not exist a thin club subset of $P_{\omega_{1}}\left(\omega_{2}\right)$.

Proof. Let $F: \omega_{2}^{<\omega} \rightarrow \omega_{2}$ be a function such that any $a$ in $P_{\omega_{1}}\left(\omega_{2}\right)$ closed under $F$ is in $C$. Let $Z$ be the stationary set of $\alpha$ in $\omega_{2} \cap \operatorname{cof}(\omega)$ closed under $F$. For each $\alpha$ in $Z$ fix a countable set $M_{\alpha} \subseteq \alpha$ such that $\sup \left(M_{\alpha}\right)=\alpha$ and $M_{\alpha}$ is closed under $F$. Fix a sequence $\left\langle Z_{s}, \xi_{s}: s \in{ }^{<\omega} 2\right\}$ as described in Lemma 2.6.

For each function $f: \omega \rightarrow 2$ define $b_{f}=c_{F}\left(\left\{\xi_{f \mid n}: n<\omega\right\}\right)$. Then $b_{f}$ is in $C$. Note that if $n<\omega$ and $\alpha$ is in $Z_{f \mid n}$, then $c_{F}\left(\left\{\xi_{f \mid m}: m \leq n\right\}\right) \subseteq M_{\alpha}$. For by Lemma 2.6(2), for $m \leq n, Z_{f \nmid n} \subseteq Z_{f \nmid m}$. So $\alpha$ is in $Z_{f \nmid m}$, and hence $\xi_{f \nmid m}$ is in $M_{\alpha}$ by (3). But $M_{\alpha}$ is closed under $F$.

Let $A=\operatorname{cl}_{F}\left(\left\{\xi_{s}: s \in{ }^{<\omega_{2}}\right\}\right)$. Since ${ }^{<\omega_{2}}$ has size $\omega, A$ is countable, and clearly each $b_{f}$ is a subset of $A$. We claim that for distinct $f$ and $g, b_{f} \neq b_{g}$. Let $n<\omega$ be least such that $f(n) \neq g(n)$. If $b_{f}=b_{g}$, then there is $k>n$ such that $\xi_{g \uparrow(n+1)}$ is in $c l_{F}\left(\left\{\xi_{f \uparrow m}: m \leq k\right\}\right)$. Fix $\alpha$ in $Z_{f \nmid k}$. By the last paragraph, $\xi_{g \uparrow(n+1)}$ is in $M_{\alpha}$. But $\alpha$ is in $Z_{f \uparrow(n+1)}$ by (2), which contradicts (4).

Let $\kappa$ be an uncountable cardinal. The Weak Reflection Principle at $\kappa$ is the statement that whenever $S$ is a stationary subset of $P_{\omega_{1}}(\kappa)$, there is a set $Y$ in $P_{\omega_{2}}(\kappa)$ such that $\omega_{1} \subseteq Y$ and $S \cap P_{\omega_{1}}(Y)$ is stationary in $P_{\omega_{1}}(Y)$. Martin's Maximum implies the Weak Reflection Principle holds for all uncountable cardinals $\kappa$ [4]. The Weak Reflection Principle at $\omega_{2}$ is equivalent to the statement that for every stationary set $S \subseteq P_{\omega_{1}}\left(\omega_{2}\right)$, there is a stationary set of uncountable $\beta<\omega_{2}$ such that $S \cap P_{\omega_{1}}(\beta)$ is stationary in $P_{\omega_{1}}(\beta)$. This is equivalent to the statement that every local club subset of $P_{\omega_{1}}\left(\omega_{2}\right)$ contains a club. The Weak Reflection Principle at $\omega_{2}$ is equiconsistent with a weakly compact cardinal 3 . 
Corollary 2.8. Suppose CH fails and there is a special Aronszajn tree on $\omega_{2}$. Then the Weak Reflection Principle at $\omega_{2}$ fails.

Proof. By Theorems 2.3 and 2.7, there is a thin local club subset of $P_{\omega_{1}}\left(\omega_{2}\right)$ which is not club. Hence the Weak Reflection Principle at $\omega_{2}$ fails.

In Sections 4 and 5 we describe models in which there is no thin stationary subset of $P_{\omega_{1}}\left(\omega_{2}\right)$. On the other hand S. Friedman proved there always exists a thin cofinal set.

Theorem 2.9 (Friedman). There exists a thin cofinal subset of $P_{\omega_{1}}\left(\omega_{2}\right)$.

Proof. We construct by induction a sequence $\left\langle S_{\alpha}: \omega_{1} \leq \alpha<\omega_{2}\right\rangle$ satisfying the properties: (1) each $S_{\alpha}$ is a cofinal subset of $P_{\omega_{1}}(\alpha)$ with size $\omega_{1},(2)$ for uncountable $\beta<\gamma$, if $a$ is in $S_{\gamma}$, then $a \cap \beta$ is in $\bigcup\left\{S_{\alpha}: \omega_{1} \leq \alpha \leq \beta\right\}$, and (3) if $\beta<\gamma<\omega_{2}, a$ is in $P_{\omega_{1}}(\gamma)$, and $a \cap \beta$ is in $S_{\beta}$, then there is $b$ in $S_{\gamma}$ such that $a \subseteq b$ and $a \cap \beta=b \cap \beta$.

Let $S_{\omega_{1}}=\omega_{1}$. Given $S_{\alpha}$, let $S_{\alpha+1}$ be the collection $\left\{b \cup\{\alpha\}: b \in S_{\alpha}\right\}$. Conditions (1), (2), and (3) follow by induction. Suppose $\gamma<\omega_{2}$ is an uncountable limit ordinal and $S_{\alpha}$ is defined for all uncountable $\alpha<\gamma$. If $\operatorname{cf}(\gamma)=\omega_{1}$, then let $S_{\gamma}=\bigcup\left\{S_{\alpha}: \omega_{1} \leq \alpha<\gamma\right\}$. The required conditions follow by induction.

Assume $\operatorname{cf}(\gamma)=\omega$. Fix an increasing sequence of uncountable ordinals $\left\langle\gamma_{n}: n<\omega\right\rangle$ unbounded in $\gamma$. Let $T_{\gamma}$ be some cofinal subset of $P_{\omega_{1}}(\gamma)$ with size $\omega_{1}$. Fix $n<\omega$. For each $x$ in $T_{\gamma}$ and $a$ in $S_{\gamma_{n}}$ define a set $b(a, x, n)$ in $P_{\omega_{1}}(\gamma)$ inductively as follows. Let $b(a, x, n) \cap \gamma_{n}=a$. Given $b(a, x, n) \cap \gamma_{m}$ in $S_{\gamma_{m}}$ for some $m \geq n$, apply condition (3) to $\gamma_{m}, \gamma_{m+1}$, and the set

$$
\left(b(a, x, n) \cap \gamma_{m}\right) \cup\left(x \cap\left[\gamma_{m}, \gamma_{m+1}\right)\right)
$$

to find $y$ in $S_{\gamma_{m+1}}$ such that $y \cap \gamma_{m}=b(a, x, n) \cap \gamma_{m}$ and $x \cap\left[\gamma_{m}, \gamma_{m+1}\right) \subseteq y$. Let $b(a, x, n) \cap \gamma_{m+1}=y$. This completes the definition of $b(a, x, n)$. Clearly $b(a, x, n) \cap \gamma_{n}=a, x \backslash \gamma_{n} \subseteq b(a, x, n)$, and for all $k \geq n, b(a, x, n) \cap \gamma_{k}$ is in $S_{\gamma_{k}}$.

Now define $S_{\gamma}=\left\{b(a, x, n): n<\omega, a \in S_{\gamma_{n}}, x \in T_{\gamma}\right\}$. We verify conditions (1), (2), and (3). Clearly $S_{\gamma}$ has size $\omega_{1}$. Let $\beta<\gamma$ and consider $b(a, x, n)$ in $S_{\gamma}$. Fix $k>n$ such that $\beta<\gamma_{k}$. Then $b(a, x, n) \cap \gamma_{k}$ is in $S_{\gamma_{k}}$. So by induction $b(a, x, n) \cap \beta$ is in $\bigcup\left\{S_{\alpha}: \omega_{1} \leq \alpha \leq \beta\right\}$. Now assume $a$ is in $P_{\omega_{1}}(\gamma), \beta<\gamma$, and $a \cap \beta$ is in $S_{\beta}$. Choose $x$ in $T_{\gamma}$ such that $a \subseteq x$. Fix $k$ such that $\beta<\gamma_{k}$. By the induction hypothesis there is $a^{\prime}$ in $S_{\gamma_{k}}$ such that $a \cap \gamma_{k} \subseteq a^{\prime}$ and $a^{\prime} \cap \beta=a \cap \beta$. Let $c=b\left(a^{\prime}, x, k\right)$. Then $c$ is in $S_{\gamma}, c \cap \beta=\left(c \cap \gamma_{k}\right) \cap \beta=a^{\prime} \cap \beta=a \cap \beta$, and $a \subseteq c$.

To prove $S_{\gamma}$ is cofinal consider $a$ in $P_{\omega_{1}}(\gamma)$. Fix $x$ in $T_{\gamma}$ such that $a \subseteq x$. By induction $S_{\gamma_{0}}$ is cofinal in $P_{\omega_{1}}\left(\gamma_{0}\right)$. So let $y$ be in $S_{\gamma_{0}}$ such that $x \cap \gamma_{0} \subseteq y$. Then $a$ is a subset of $b(y, x, 0)$.

Now define $S=\bigcup\left\{S_{\beta}: \omega_{1} \leq \beta<\omega_{2}\right\}$. Conditions (1) and (2) imply that $S$ is thin and cofinal in $P_{\omega_{1}}\left(\omega_{2}\right)$.

\section{DisJoint Club SEQUenCES}

We introduce a combinatorial property of $\omega_{2}$ which implies there does not exist a thin stationary subset of $P_{\omega_{1}}\left(\omega_{2}\right)$. This property follows from Martin's Maximum and is equiconsistent with a Mahlo cardinal. It implies there exists a fat stationary subset of $\omega_{2}$ which cannot acquire a club subset by any forcing poset which preserves $\omega_{1}$ and $\omega_{2}$. 
Definition 3.1. A disjoint club sequence on $\omega_{2}$ is a sequence $\left\langle\mathcal{C}_{\alpha}: \alpha \in A\right\rangle$ such that $A$ is a stationary subset of $\omega_{2} \cap \operatorname{cof}\left(\omega_{1}\right)$, each $\mathcal{C}_{\alpha}$ is a club subset of $P_{\omega_{1}}(\alpha)$, and $\mathcal{C}_{\alpha} \cap \mathcal{C}_{\beta}$ is empty for all $\alpha<\beta$ in $A$.

Proposition 3.2. Suppose there is a disjoint club sequence on $\omega_{2}$. Then there does not exist a thin stationary subset of $P_{\omega_{1}}\left(\omega_{2}\right)$.

Proof. Let $\left\langle\mathcal{C}_{\alpha}: \alpha \in A\right\rangle$ be a disjoint club sequence. Suppose for a contradiction there exists a thin stationary set. By Lemma 2.2 fix a thin stationary set $T \subseteq$ $P_{\omega_{1}}\left(\omega_{2}\right)$ such that for all uncountable $\beta<\omega_{2}, T \cap P_{\omega_{1}}(\beta)$ is stationary in $P_{\omega_{1}}(\beta)$. Then for each $\beta$ in $A$ we can choose a set $a_{\beta}$ in $\mathcal{C}_{\beta} \cap T$. Since $\operatorname{cf}(\beta)=\omega_{1}, \sup \left(a_{\beta}\right)<\beta$. By Fodor's Lemma there is a stationary set $B \subseteq A$ and a fixed $\gamma<\omega_{2}$ such that for all $\beta$ in $B, \sup \left(a_{\beta}\right)=\gamma$. If $\alpha<\beta$ are in $B$, then $a_{\alpha} \neq a_{\beta}$ since $\mathcal{C}_{\alpha} \cap \mathcal{C}_{\beta}$ is empty. So the set $\left\{a_{\beta}: \beta \in B\right\}$ witnesses that $T$ is not thin, which is a contradiction.

Lemma 3.3. Suppose there is a disjoint club sequence $\left\langle\mathcal{C}_{\alpha}: \alpha \in A\right\rangle$ on $\omega_{2}$. Let $W$ be an outer model with the same $\omega_{1}$ and $\omega_{2}$ in which $A$ is still stationary. Then there is a disjoint club sequence $\left\langle\mathcal{D}_{\alpha}: \alpha \in A\right\rangle$ in $W$.

Proof. By the proof of Lemma 2.4, each $\mathcal{C}_{\alpha}$ contains a club set $\mathcal{D}_{\alpha}$ in $W$. Since $\omega_{1}$ is preserved, each $\alpha$ in $A$ still has cofinality $\omega_{1}$.

Theorem 3.4. Suppose $\left\langle\mathcal{C}_{\alpha}: \alpha \in A\right\rangle$ is a disjoint club sequence on $\omega_{2}$. Then $A \cup \operatorname{cof}(\omega)$ does not contain a club.

Proof. Suppose for a contradiction that $A \cup \operatorname{cof}(\omega)$ contains a club. Without loss of generality $2^{\omega_{1}}=\omega_{2}$. Otherwise work in a generic extension $W$ by $\operatorname{CoLL}\left(\omega_{2}, 2^{\omega_{1}}\right)$ : In $W$ the set $A \cup \operatorname{cof}(\omega)$ contains a club, and by Lemma 3.3 there is a disjoint club sequence $\left\langle\mathcal{D}_{\alpha}: \alpha \in A\right\rangle$.

Since $2^{\omega_{1}}=\omega_{2}, H\left(\omega_{2}\right)$ has size $\omega_{2}$. Fix a bijection $h: H\left(\omega_{2}\right) \rightarrow \omega_{2}$. Let $\mathcal{A}$ denote the structure $\left\langle H\left(\omega_{2}\right), \in, h\right\rangle$. Define $B$ as the set of $\alpha$ in $\omega_{2} \cap \operatorname{cof}\left(\omega_{1}\right)$ such that there exists an increasing and continuous sequence $\left\langle N_{i}: i<\omega_{1}\right\rangle$ of countable elementary substructures of $\mathcal{A}$ such that:

(1) for $i<\omega_{1}, N_{i}$ is in $N_{i+1}$,

(2) the set $\left\{N_{i} \cap \omega_{2}: i<\omega_{1}\right\}$ is club in $P_{\omega_{1}}(\alpha)$.

We claim that $B$ is stationary in $\omega_{2}$. To prove this let $C \subseteq \omega_{2}$ be club. Let $\mathcal{B}$ be the expansion of $\mathcal{A}$ by the function $\alpha \mapsto \min (C \backslash \alpha)$. Define by induction an increasing and continuous sequence $\left\langle N_{i}: i<\omega_{1}\right\rangle$ of elementary substructures of $\mathcal{B}$ such that for all $i<\omega_{1}, N_{i}$ is in $N_{i+1}$. Let $N=\bigcup\left\{N_{i}: i<\omega_{1}\right\}$. Then $\omega_{1} \subseteq N$ so $N \cap \omega_{2}$ is an ordinal. Write $\alpha=N \cap \omega_{2}$. Then $\alpha$ is in $C$ and $\left\{N_{i} \cap \omega_{2}: i<\omega_{1}\right\}$ is club in $P_{\omega_{1}}(\alpha)$. So $\alpha$ is in $B \cap C$.

Since $A \cup \operatorname{cof}(\omega)$ contains a club, $A \cap B$ is stationary. For each $\alpha$ in $A \cap B$ fix a sequence $\left\langle N_{i}^{\alpha}: i<\omega_{1}\right\rangle$ as described in the definition of $B$. Then $\left\{N_{i}^{\alpha} \cap \omega_{2}\right.$ : $\left.i<\omega_{1}\right\} \cap \mathcal{C}_{\alpha}$ is club in $P_{\omega_{1}}(\alpha)$. So there exists a club set $c_{\alpha} \subseteq \omega_{1}$ such that $\left\{N_{i}^{\alpha} \cap \omega_{2}: i \in c_{\alpha}\right\}$ is club and is a subset of $\mathcal{C}_{\alpha}$. Write $i_{\alpha}=\min \left(c_{\alpha}\right)$ and let $d_{\alpha}=c_{\alpha} \backslash\left\{i_{\alpha}\right\}$.

Define $S=\left\{N_{i}^{\alpha}: \alpha \in A \cap B, i \in d_{\alpha}\right\}$. If $N$ is in $S$, then there is a unique pair $\alpha$ in $A \cap B$ and $i$ in $d_{\alpha}$ such that $N=N_{i}^{\alpha}$. For if $N=N_{i}^{\alpha}=N_{j}^{\beta}$, then $N \cap \omega_{2}$ is in $\mathcal{C}_{\alpha} \cap \mathcal{C}_{\beta}$, so $\alpha=\beta$. Clearly then $i=j$. Also note that if $N_{i}^{\alpha}$ is in $S$, then $N_{i_{\alpha}}^{\alpha}$ is in $N_{i}^{\alpha}$. So the function $H: S \rightarrow H\left(\omega_{2}\right)$ defined by $H\left(N_{i}^{\alpha}\right)=N_{i_{\alpha}}^{\alpha}$ is well defined and regressive. 
We claim that $S$ is stationary in $P_{\omega_{1}}\left(H\left(\omega_{2}\right)\right)$. To prove this let $F: H\left(\omega_{2}\right)^{<\omega} \rightarrow$ $H\left(\omega_{2}\right)$ be a function. Define $G: \omega_{2}^{<\omega} \rightarrow \omega_{2}$ by letting $G\left(\alpha_{0}, \ldots, \alpha_{n}\right)$ be equal to $h\left(F\left(h^{-1}\left(\alpha_{0}\right), \ldots, h^{-1}\left(\alpha_{n}\right)\right)\right)$. Let $E$ be the club set of $\alpha$ in $\omega_{2}$ closed under $G$. Fix $\alpha$ in $E \cap A \cap B$. Then there is $i$ in $d_{\alpha}$ such that $N_{i}^{\alpha} \cap \omega_{2}$ is closed under $G$. We claim that $N_{i}^{\alpha}$ is closed under $F$. Given $a_{0}, \ldots, a_{n}$ in $N_{i}^{\alpha}$, the ordinals $h\left(a_{0}\right), \ldots, h\left(a_{n}\right)$ are in $N_{i}^{\alpha} \cap \omega_{2}$. So $\gamma=G\left(h\left(a_{0}\right), \ldots, h\left(a_{n}\right)\right)=h\left(F\left(a_{0}, \ldots, a_{n}\right)\right)$ is in $N_{i}^{\alpha} \cap \omega_{2}$. Therefore $h^{-1}(\gamma)=F\left(a_{0}, \ldots, a_{n}\right)$ is in $N_{i}^{\alpha}$.

Since $S$ is stationary and $H: S \rightarrow H\left(\omega_{2}\right)$ is regressive, there is a stationary set $S^{*} \subseteq S$ and a fixed $N$ such that for all $N_{i}^{\alpha}$ in $S^{*}, H\left(N_{i}^{\alpha}\right)=N$. The set $S^{*}$, being stationary, must have size $\omega_{2}$. So there are distinct $\alpha$ and $\beta$ such that for some $i$ in $d_{\alpha}$ and $j$ in $d_{\beta}, N_{i}^{\alpha}$ and $N_{j}^{\beta}$ are in $S^{*}$. Then $N=N_{i_{\alpha}}^{\alpha}=N_{i_{\beta}}^{\beta}$. So $N \cap \omega_{2}$ is in $\mathcal{C}_{\alpha} \cap \mathcal{C}_{\beta}$, which is a contradiction.

Abraham and Shelah [1] asked the following question: Assume that $A$ is a stationary subset of $\omega_{2} \cap \operatorname{cof}\left(\omega_{1}\right)$. Does there exist an $\omega_{1}$-distributive forcing poset which adds a club subset to $A \cup \operatorname{cof}(\omega)$ ? We answer this question in the negative.

Corollary 3.5. Assume that $\left\langle\mathcal{C}_{\alpha}: \alpha \in A\right\rangle$ is a disjoint club sequence. Let $W$ be an outer model of $V$ with the same $\omega_{1}$ and $\omega_{2}$. Then in $W, A \cup \operatorname{cof}(\omega)$ does not contain a club subset.

Proof. If $A$ remains stationary in $W$, then by Lemma 3.3 there is a disjoint club sequence $\left\langle\mathcal{D}_{\alpha}: \alpha \in A\right\rangle$ in $W$. By Theorem 3.4 $A \cup \operatorname{cof}(\omega)$ does not contain a club in $W$.

\section{Martin's Maximum}

In this section we prove that Martin's Maximum implies there exists a disjoint club sequence on $\omega_{2}$. We apply MM to the poset for adding a Cohen real and then forcing a continuous $\omega_{1}$-chain through $P_{\omega_{1}}\left(\omega_{2}\right) \backslash V$.

Theorem 4.1 (Krueger). Martin's Maximum implies there exists a disjoint club sequence on $\omega_{2}$.

We will use the following theorem from [1].

Theorem 4.2. Suppose $\mathbb{P}$ is $\omega_{1}$-c.c. and adds a real. Then $\mathbb{P}$ forces that $\left(P_{\omega_{1}}\left(\omega_{2}\right) \backslash\right.$ $V)$ is stationary in $P_{\omega_{1}}\left(\omega_{2}\right)$.

Note: Gitik [6] proved that the conclusion of Theorem 4.2 holds for any outer model of $V$ which contains a new real and computes the same $\omega_{1}$.

Suppose that $S$ is a stationary subset of $P_{\omega_{1}}\left(\omega_{2}\right)$. Following [3] we define a forcing poset $\mathbb{P}(S)$ which adds a continuous $\omega_{1}$-chain through $S$. A condition in $\mathbb{P}(S)$ is a countable increasing and continuous sequence $\left\langle a_{i}: i \leq \beta\right\rangle$ of elements from $S$, where for each $i<\beta, a_{i} \cap \omega_{1}<a_{i+1} \cap \omega_{1}$. The ordering on $\mathbb{P}(S)$ is by extension of sequences.

Proposition 4.3. If $S \subseteq P_{\omega_{1}}\left(\omega_{2}\right)$ is stationary, then $\mathbb{P}(S)$ is $\omega$-distributive.

Proof. Suppose $p$ forces $\dot{f}: \omega \rightarrow$ On. Let $\theta \gg \omega_{2}$ be a regular cardinal such that $\dot{f}$ is in $H(\theta)$. Since $S$ is stationary, we can fix a countable elementary substructure $N$ of the model

$$
\langle H(\theta), \in, S, \mathbb{P}(S), p, \dot{f}\rangle
$$


such that $N \cap \omega_{2}$ is in $S$. Let $\left\langle D_{n}: n<\omega\right\rangle$ be an enumeration of all the dense subsets of $\mathbb{P}(S)$ in $N$. Inductively define a decreasing sequence $\left\langle p_{n}: n<\omega\right\rangle$ of elements of $N \cap \mathbb{P}$ such that $p_{0}=p$ and $p_{n+1}$ is a refinement of $p_{n}$ in $D_{n} \cap N$. Write $\bigcup\left\{p_{n}: n<\omega\right\}=\left\langle b_{i}: i<\gamma\right\rangle$. Clearly $\bigcup\left\{b_{i}: i<\gamma\right\}=N \cap \omega_{2}$. Since $N \cap \omega_{2}$ is in $S$, the sequence $\left\langle b_{i}: i<\gamma\right\rangle \cup\left\{\left\langle\gamma, N \cap \omega_{2}\right\rangle\right\}$ is a condition below $p$ which decides $\dot{f}(n)$ for all $n<\omega$.

Theorem 4.4. Suppose $\mathbb{P}$ is an $\omega_{1}$-c.c. forcing poset which adds a real. Let $\dot{S}$ be a name such that $\mathbb{P}$ forces $\dot{S}=\left(P_{\omega_{1}}\left(\omega_{2}\right) \backslash V\right)$. Then $\mathbb{P} * \mathbb{P}(\dot{S})$ preserves stationary subsets of $\omega_{1}$.

Proof. By Theorem 4.2 and Proposition 4.3 , the poset $\mathbb{P} * \mathbb{P}(\dot{S})$ preserves $\omega_{1}$. Let $A$ be a stationary subset of $\omega_{1}$ in $V$. Suppose $p * \dot{q}$ is a condition in $\mathbb{P} * \mathbb{P}(\dot{S})$ which forces that $\dot{C}$ is a club subset of $\omega_{1}$.

Let $G$ be a generic filter for $\mathbb{P}$ over $V$ which contains $p$. In $V[G]$ fix a regular cardinal $\theta \gg \omega_{2}$ and let

$$
\mathcal{A}=\langle H(\theta), \in, A, S, q, \dot{C}\rangle .
$$

Fix a Skolem function $F: H(\theta)^{<\omega} \rightarrow H(\theta)$ for $\mathcal{A}$. Define $F^{*}: \omega_{2}^{<\omega} \rightarrow P_{\omega_{1}}\left(\omega_{2}\right)$ by letting

$$
F^{*}\left(\alpha_{0}, \ldots, \alpha_{n}\right)=c l_{F}\left(\left\{\alpha_{0}, \ldots, \alpha_{n}\right\}\right) \cap \omega_{2} .
$$

Since $\mathbb{P}$ is $\omega_{1}$-c.c. there is a function $H: \omega_{2}^{<\omega} \rightarrow P_{\omega_{1}}\left(\omega_{2}\right)$ in $V$ such that for all $\vec{\alpha}$ in $\omega_{2}^{<\omega}, F^{*}(\vec{\alpha}) \subseteq H(\vec{\alpha})$. Let $Z^{*}$ be the stationary set of $\alpha$ in $\omega_{2} \cap \operatorname{cof}(\omega)$ closed under $H$.

Working in $V$, since $A$ is stationary we can fix for each $\alpha$ in $Z^{*}$ a countable cofinal set $M_{\alpha} \subseteq \alpha$ closed under $H$ with $M_{\alpha} \cap \omega_{1}$ in $A$. By Fodor's Lemma there is $Z \subseteq Z^{*}$ stationary and $\delta$ in $A$ such that for all $\alpha$ in $Z, M_{\alpha} \cap \omega_{1}=\delta$. Fix a sequence $\left\langle\xi_{s}, Z_{s}: s \in{ }^{<\omega} 2\right\rangle$ satisfying conditions (1)-(4) of Lemma 2.6 .

Let $f: \omega \rightarrow 2$ be a function in $V[G] \backslash V$. For each $n<\omega$ let $M_{n}$ denote $c l_{H}\left(\delta \cup\left\{\xi_{f \nmid m}: m \leq n\right\}\right)$. Define $M=\bigcup\left\{M_{n}: n<\omega\right\}$. Note that $M$ is closed under $H$ and hence it is closed under $F^{*}$. Therefore $N=\operatorname{cl}_{F}(M)$ is an elementary substructure of $\mathcal{A}$ such that $N \cap \omega_{2}=M$.

As in the proof of Theorem 2.7, for all $n<\omega$, if $\alpha$ is in $Z_{f i n}$ then $M_{n} \subseteq M_{\alpha}$. Note that $M \cap \omega_{1}=\delta$. For if $\gamma$ is in $M \cap \omega_{1}$, there is $n<\omega$ such that $\gamma$ is in $M_{n}$. Fix $\alpha$ in $Z_{f \nmid n}$. Then $\gamma$ is in $M_{\alpha} \cap \omega_{1}=\delta$.

We prove that $M$ is not in $V$ by showing how to compute $f$ by induction from $M$. Suppose $f\left\lceil n\right.$ is known. Fix $j<2$ such that $f(n) \neq j$. We claim that $\xi_{\left(f\lceil n)^{\wedge} j\right.}$ is not in $M$. Otherwise there is $k>n$ such that $\xi_{(f \nmid n)^{\wedge} j}$ is in $M_{k}$. Fix $\alpha$ in $Z_{f \nmid k}$. Then $\xi_{\left(f\lceil n)^{\wedge} j\right.}$ is in $M_{\alpha}$. But $\alpha$ is in $Z_{f \uparrow(n+1)}$, contradicting Lemma 2.6(4). So $f(n)$ is the unique $i<2$ such that $\xi_{(f \mid n)^{\wedge} i}$ is in $M$. This completes the definition of $f$ from $M$. Since $f$ is not in $V$, neither is $M$.

Let $\left\langle D_{n}: n<\omega\right\rangle$ enumerate the dense subsets of $\mathbb{P}(S)$ lying in $N$. Inductively define a decreasing sequence $\left\langle q_{n}: n<\omega\right\rangle$ in $N \cap \mathbb{P}(S)$ such that $q_{0}=q$ and $q_{n+1}$ is in $D_{n} \cap N$. Write $\bigcup\left\{q_{n}: n<\omega\right\}=\left\langle b_{i}: i<\gamma\right\rangle$. Clearly $\bigcup\left\{b_{i}: i<\gamma\right\}=N \cap \omega_{2}=M$, and since $M$ is not in $V, r=\left\langle b_{i}: i<\gamma\right\rangle \cup\{\langle\gamma, M\rangle\}$ is a condition in $\mathbb{P}(S)$. By an easy density argument, $r$ forces that $N \cap \omega_{1}=\delta$ is a limit point of $\dot{C}$, and hence is in $\dot{C}$. Let $\dot{r}$ be a name for $r$. Then $p * \dot{r} \leq p * \dot{q}$ and $p * \dot{r}$ forces that $\delta$ is in $A \cap \dot{C}$. 
Now we are ready to prove that MM implies there exists a disjoint club sequence on $\omega_{2}$.

Proof of Theorem 4.1. Assume Martin's Maximum. Inductively define $A$ and $\left\langle\mathcal{C}_{\alpha}: \alpha \in A\right\rangle$ as follows. Suppose $\alpha$ is in $\omega_{2} \cap \operatorname{cof}\left(\omega_{1}\right)$ and $A \cap \alpha$ and $\left\langle\mathcal{C}_{\beta}: \beta \in A \cap \alpha\right\rangle$ are defined. Let $\alpha$ be in $A$ iff the set $\bigcup\left\{\mathcal{C}_{\beta}: \beta \in A \cap \alpha\right\}$ is non-stationary in $P_{\omega_{1}}(\alpha)$. If $\alpha$ is in $A$, then choose a club set $\mathcal{C}_{\alpha} \subseteq P_{\omega_{1}}(\alpha)$ with size $\omega_{1}$ which is disjoint from this union.

This completes the definition. We prove that $A$ is stationary. Then clearly $\left\langle\mathcal{C}_{\alpha}: \alpha \in A\right\rangle$ is a disjoint club sequence. Fix a club set $C \subseteq \omega_{2}$.

Let ADD denote the forcing poset for adding a single Cohen real with finite conditions and let $\dot{S}$ be an ADD-name for the set $\left(P_{\omega_{1}}\left(\omega_{2}\right) \backslash V\right)$. By Theorem 4.4 the poset $\mathrm{ADD} * \mathbb{P}(\dot{S})$ preserves stationary subsets of $\omega_{1}$. We will apply Martin's Maximum to this poset after choosing a suitable collection of dense sets.

For each $\alpha<\omega_{2}$ fix a surjection $f_{\alpha}: \omega_{1} \rightarrow \alpha$. If $\beta$ is in $A$ enumerate $\mathcal{C}_{\beta}$ as $\left\langle a_{i}^{\beta}: i<\omega_{1}\right\rangle$. For every quadruple $i, j, k, l$ of countable ordinals let $D(i, j, k, l)$ denote the set of conditions $p * \dot{q}$ such that:

(1) $p$ forces that $i$ and $j$ are in the domain of $\dot{q}$, and for some $\beta_{i}$ and $\beta_{j}, p$ forces $\beta_{i}=\sup (\dot{q}(i))$ and $\beta_{j}=\sup (\dot{q}(j))$,

(2) there is some $\zeta<\omega_{1}$ such that $p$ forces $\zeta$ is the least element in $\operatorname{dom}(\dot{q})$ such that $f_{\beta_{i}}(j) \in \dot{q}(\zeta)$,

(3) there is $\xi$ in $C$ larger than $\beta_{i}$ and $\beta_{j}$ such that $p$ forces $\xi$ is the supremum of the maximal set in $\dot{q}$,

(4) if $f_{\beta_{j}}(k)=\gamma$ is in $A$, then there is $z$ such that $p$ forces $z$ is in the symmetric difference $\dot{q}(i) \triangle a_{l}^{\gamma}$.

It is routine to check that $D(i, j, k, l)$ is dense.

Let $G * H$ be a filter on $\operatorname{ADD} * \mathbb{P}(\dot{S})$ intersecting each $D(i, j, k, l)$. For $i<\omega_{1}$ define $a_{i}$ as the set of $\beta$ for which there exists some $p * \dot{q}$ in $G * H$ such that $p$ forces $i \in \operatorname{dom}(\dot{q})$ and $p$ forces $\beta$ is in $\dot{q}(i)$. The definition of the dense sets implies that $\left\langle a_{i}: i\left\langle\omega_{1}\right\rangle\right.$ is increasing, continuous, and cofinal in $P_{\omega_{1}}(\alpha)$ for some $\alpha$ in $C \cap \operatorname{cof}\left(\omega_{1}\right)$. By (4), for each $\gamma$ in $A \cap \alpha,\left\{a_{i}: i<\omega_{1}\right\}$ is disjoint from $\mathcal{C}_{\gamma}$. Therefore $\bigcup\left\{\mathcal{C}_{\gamma}: \gamma \in A \cap \alpha\right\}$ is non-stationary in $P_{\omega_{1}}(\alpha)$, hence by the definition of $A, \alpha$ is in $A \cap C$. So $A$ is stationary.

\section{The EQUiCOnSISTENCY RESUlt}

We now prove Theorem 0.1 establishing the consistency strength of each of the following statements to be exactly a Mahlo cardinal: (1) There does not exist a thin stationary subset of $P_{\omega_{1}}\left(\omega_{2}\right)$. (2) There exists a disjoint club sequence on $\omega_{2}$. (3) There exists a fat stationary set $S \subseteq \omega_{2}$ such that any forcing poset which preserves $\omega_{1}$ and $\omega_{2}$ does not add a club subset to $S$.

By [5] if there exists a thin stationary subset of $P_{\omega_{1}}\left(\omega_{2}\right)$, then for any fat stationary set $S \subseteq \omega_{2}$, there is a forcing poset which preserves cardinals and adds a club subset to $S$. So (2) and (3) both imply (1), which in turn implies there is no special Aronszajn tree on $\omega_{2}$. So $\omega_{2}$ is Mahlo in $L$ by 8 .

In the other direction assume that $\kappa$ is a Mahlo cardinal. We will define a revised countable support iteration which collapses $\kappa$ to become $\omega_{2}$ and adds a disjoint club sequence on $\omega_{2}$. At individual stages of the iteration we force with either a collapse forcing or the poset $\mathrm{ADD} * \mathbb{P}(\dot{S})$ from the previous section. To ensure that $\omega_{1}$ is 
not collapsed we verify that $\mathrm{ADD} * \mathbb{P}(\dot{S})$ satisfies an iterable condition known as the $\mathbb{I}$-universal property. Our description of this construction is self-contained, except for the proof of Theorem 5.9 which summarizes the relevant properties of the RCS iteration. For more information on such iterations and the $\mathbb{I}$-universal property see [10.

Definition 5.1. A pair $\langle T, \mathbf{I}\rangle$ is a tagged tree if:

(1) $T \subseteq{ }^{<\omega}$ On is a tree such that each $\eta$ in $T$ has at least one successor,

(2) $\mathbf{I}: T \rightarrow V$ is a partial function such that each $\mathbf{I}(\eta)$ is an ideal on some set $X_{\eta}$ and for each $\eta$ in the domain of $\mathbf{I}$, the set $\left\{\alpha: \eta^{\wedge} \alpha \in T\right\}$ is in $(\mathbf{I}(\eta))^{+}$,

(3) for each cofinal branch $b$ of $T$, there are infinitely many $n<\omega$ such that $b\lceil n$ is in the domain of $\mathbf{I}$.

If $\eta$ is in the domain of $\mathbf{I}$, we say that $\eta$ is a splitting point of $T$. It follows from (1) and (3) that for every $\eta$ in $T$ there is $\eta \triangleleft \nu$ which is a splitting point.

Definition 5.2. Let $\mathbb{I}$ be a family of ideals and $\langle T, \mathbf{I}\rangle$ a tagged tree. Then $\langle T, \mathbf{I}\rangle$ is an $\mathbb{I}$-tree if for each splitting point $\eta$ in $T, \mathbf{I}(\eta)$ is in $\mathbb{I}$.

Suppose $T \subseteq{ }^{<\omega}$ On is a tree. If $\eta$ is in $T$, let $T^{[\eta]}$ denote the tree $\{\nu \in T$ : $\nu \unlhd \eta$ or $\eta \unlhd \nu\}$. A set $J \subseteq T$ is called a front if for distinct nodes in $J$, neither is an initial segment of the other, and for any cofinal branch $b$ of $T$ there is $\eta$ in $J$ which is an initial segment of $b$.

Definition 5.3. Suppose $\langle T, \mathbf{I}\rangle$ is tagged tree. Let $\theta$ be a regular cardinal such that $\langle T, \mathbf{I}\rangle$ is in $H(\theta)$, and let $<_{\theta}$ be a well-ordering of $H(\theta)$. A sequence $\left\langle N_{\eta}: \eta \in T\right\rangle$ is a tree of models for $\theta$ provided that:

(1) each $N_{\eta}$ is a countable elementary substructure of $\left\langle H(\theta), \in,<_{\theta},\langle T, \mathbf{I}\rangle\right\rangle$,

(2) if $\eta \triangleleft \nu$ in $T$, then $N_{\eta} \prec N_{\nu}$,

(3) for each $\eta$ in $T, \eta$ is in $N_{\eta}$.

Definition 5.4. Suppose $\langle T, \mathbf{I}\rangle$ is an $\mathbb{I}$-tree, and $\theta$ is a regular cardinal such that $H(\theta)$ contains $\langle T, \mathbf{I}\rangle$ and $\mathbb{I}$. A sequence $\left\langle N_{\eta}: \eta \in T\right\rangle$ is an $\mathbb{I}$-suitable tree of models for $\theta$ if it is a tree of models for $\theta$, and for every $\eta$ in $T$ and $I$ in $\mathbb{I} \cap N_{\eta}$, the set

$$
\left\{\nu \in T^{[\eta]}: \nu \text { is a splitting point and } \mathbf{I}(\nu)=I\right\}
$$

contains a front in $T^{[\eta]}$.

Definition 5.5. Let $\langle T, \mathbf{I}\rangle, \mathbb{I}$, and $\theta$ be as in Definition 5.4. A sequence $\left\langle N_{\eta}: \eta \in T\right\rangle$ is an $\omega_{1}$-strictly $\mathbb{I}$-suitable tree of models for $\theta$ if it is an $\mathbb{I}$-suitable tree of models for $\theta$ and there exists $\delta<\omega_{1}$ such that for all $\eta$ in $T, N_{\eta} \cap \omega_{1}=\delta$.

If $\left\langle N_{\eta}: \eta \in T\right\rangle$ is a tree of models and $b$ is a cofinal branch of $T$, write $N_{b}$ for the set $\bigcup\left\{N_{b \mid n}: n<\omega\right\}$. Note that if $\left\langle N_{\eta}: \eta \in T\right\rangle$ is an $\omega_{1}$-strictly I-suitable tree of models for $\theta$, then for any cofinal branch $b$ of $T, N_{b} \cap \omega_{1}=N_{\langle\rangle} \cap \omega_{1}$.

Lemma 5.6. Let $\langle T, \mathbf{I}\rangle, \mathbb{I}$, and $\theta$ be as in Definition 5.4, and let $\left\langle N_{\eta}: \eta \in T\right\rangle$ be an $\omega_{1}$-strictly $\mathbb{I}$-suitable tree of models for $\theta$. Suppose $\eta \triangleleft \nu$ in $T$ and $\left(N_{\nu} \cap \omega_{2}\right) \backslash N_{\eta}$ is non-empty. Let $\gamma$ be the minimum element of $\left(N_{\nu} \cap \omega_{2}\right) \backslash N_{\eta}$. Then $\gamma \geq \sup \left(N_{\eta} \cap \omega_{2}\right)$.

Proof. Otherwise there is $\beta$ in $N_{\eta} \cap \omega_{2}$ such that $\gamma<\beta$. By elementarity, there is a surjection $f: \omega_{1} \rightarrow \beta$ in $N_{\eta}$. So $f^{-1}(\gamma) \in N_{\nu} \cap \omega_{1}=N_{\eta} \cap \omega_{1}$. Hence $f\left(f^{-1}(\gamma)\right)=\gamma$ is in $N_{\eta}$, which is a contradiction. 
Let $\mathbb{I}$ be a family of ideals. We say that $\mathbb{I}$ is restriction-closed if for all $I$ in $\mathbb{I}$, for any set $A$ in $I^{+}$, the ideal $I \uparrow A$ is in $\mathbb{I}$. If $\mu$ is a regular uncountable cardinal, we say that $\mathbb{I}$ is $\mu$-complete if each ideal in $\mathbb{I}$ is $\mu$-complete.

Definition 5.7. Suppose that II is a non-empty restriction-closed $\omega_{2}$-complete family of ideals and let $\mathbb{P}$ be a forcing poset. Then $\mathbb{P}$ satisfies the $\mathbb{I}$-universal property if for all sufficiently large regular cardinals $\theta$ with $\mathbb{I}$ in $H(\theta)$, if $\left\langle N_{\eta}: \eta \in T\right\rangle$ is an $\omega_{1}$-strictly $\mathbb{I}$-suitable tree of models for $\theta$, then for all $p$ in $N_{\langle\rangle} \cap \mathbb{P}$ there is $q \leq p$ such that $q$ forces that there is a cofinal branch $b$ of $T$ such that $N_{b}[\dot{G}] \cap \omega_{1}=N_{\langle\rangle} \cap \omega_{1}$.

Definition 5.7 is Shelah's characterization of the $\mathbb{I}$-universal property given in 10, Chapter XV 2.11, 2.12, and 2.13. Note that in the definition, $q$ is semigeneric for $N_{\langle\rangle}$. In 2.12 Shelah proves that there are stationarily many structures $N$ for which $N=N_{\langle\rangle}$for some $\omega_{1}$-strictly $\mathbb{I}$-suitable tree of models $\left\langle N_{\eta}: \eta \in T\right\rangle$. So by standard arguments if $\mathbb{P}$ satisfies the $\mathbb{I}$-universal property, then $\mathbb{P}$ preserves $\omega_{1}$ and preserves stationary subsets of $\omega_{1}$. Note that any semiproper forcing poset satisfies the I-universal property.

Theorem 5.8. Let II be the family of ideals of the form $N S_{\omega_{2}} \uparrow A$, where $A$ is a stationary subset of $\omega_{2} \cap \operatorname{cof}(\omega)$. Let $\dot{S}$ be an ADD-name for the set $\left(P_{\omega_{1}}\left(\omega_{2}\right) \backslash V\right)$. Then $\operatorname{ADD} * \mathbb{P}(\dot{S})$ satisfies the $\mathbb{I}$-universal property.

Proof. Fix a regular cardinal $\theta \gg \omega_{2}$ and let $\left\langle N_{\eta}: \eta \in T\right\rangle$ be an $\omega_{1}$-strictly $\mathbb{I}$ suitable tree of models for $\theta$. Let $p * \dot{q}$ be a condition in $($ ADD $* \mathbb{P}(\dot{S})) \cap N_{\langle\rangle}$. We find a refinement of $p * \dot{q}$ which forces that there is a cofinal branch $b$ of $T$ such that $N_{b}[\dot{G} * \dot{H}] \cap \omega_{1}=N_{\langle\rangle} \cap \omega_{1}$.

We use an argument similar to the proof of Lemma 2.6 to define a sequence $\left\langle\eta_{s}, \xi_{s}: s \in<\omega 2\right\rangle$ satisfying:

(1) each $\eta_{s}$ is in $T$, each $\xi_{s}$ is in $N_{\eta_{s}} \cap \omega_{2}$, and $s \triangleleft t$ implies $\eta_{s} \triangleleft \eta_{t}$,

(2) if $\widehat{s} 0 \unlhd t$, then $\xi_{s \wedge 1}$ is not in $N_{\eta_{t}}$, and if $\widehat{s \wedge} 1 \unlhd u$, then $\xi_{s \wedge 0}$ is not in $N_{\eta_{u}}$.

Let $\eta_{\langle\rangle}=\langle\rangle$and $\xi_{\langle\rangle}=0$. Suppose $\eta_{s}$ is defined. Choose a splitting point $\nu_{s}$ in $T$ above $\eta_{s}$. Let $Z$ denote the set of $\alpha<\omega_{2}$ such that $\nu_{s} \widehat{ } \alpha$ is in $T$. Since $\nu_{s}$ is a splitting point, by the definition of $\mathbb{I}$ the set $Z$ is a stationary subset of $\omega_{2} \cap \operatorname{cof}(\omega)$. For each $\alpha$ in $Z, \alpha$ is in $N_{\left(\nu_{s} \wedge \alpha\right)}$ and has cofinality $\omega$, so $N_{\left(\nu_{s} \wedge \alpha\right)} \cap \alpha$ is a countable cofinal subset of $\alpha$. Define $X_{s}$ as the set of $\xi$ in $\omega_{2}$ such that the set

$$
\left\{\alpha \in Z: \xi \in N_{\left(\nu_{s} \wedge \alpha\right)} \cap \alpha\right\}
$$

is stationary. An easy argument using Fodor's Lemma shows that $X_{s}$ is unbounded in $\omega_{2}$. For all large enough $\alpha$ in $Z$, the set $\left(X_{s} \backslash \sup \left(N_{\nu_{s}} \cap \omega_{2}\right)\right) \cap \alpha$ has size $\omega_{1}$. So there is a stationary set $Z_{1}^{\prime} \subseteq Z$ and an ordinal $\xi_{s^{\wedge} 0}$ in $X_{s}$ such that $\xi_{s^{\wedge} 0}$ is larger than $\sup \left(N_{\nu_{s}} \cap \omega_{2}\right)$ and for all $\alpha$ in $Z_{1}^{\prime}, \xi_{s \wedge 0}$ is not in $N_{\left(\nu_{s} \wedge \alpha\right)} \cap \alpha$. Let $Z_{0}^{\prime}$ be the stationary set of $\alpha$ in $Z$ such that $\xi_{s^{\wedge} 0}$ is in $N_{\left(\nu_{s} \wedge \alpha\right)} \cap \alpha$. Now define $Y_{s}$ as the set of $\xi$ in $\omega_{2}$ such that the set

$$
\left\{\alpha \in Z_{1}^{\prime}: \xi \in N_{\left(\nu_{s} \wedge \alpha\right)} \cap \alpha\right\}
$$

is stationary. Again we can find $Z_{0} \subseteq Z_{0}^{\prime}$ stationary and $\xi_{s \wedge 1}$ in $Y_{s}$ such that $\xi_{s^{\wedge} 1}$ is larger than $\sup \left(N_{\nu_{s}} \cap \omega_{2}\right)$ and for all $\alpha$ in $Z_{0}, \xi_{s \wedge 1}$ is not in $N_{\left(\nu_{s} \wedge \alpha\right)} \cap \alpha$. Let $Z_{1}$ be the stationary set of $\alpha$ in $Z_{1}^{\prime}$ such that $\xi_{s \wedge 1}$ is in $N_{\left(\nu_{s} \wedge \alpha\right)} \cap \alpha$.

Now define $\eta_{s^{\wedge} 0}$ to be equal to $\nu_{s} \wedge \alpha$ for some $\alpha$ in $Z_{0}$ larger than $\xi_{s^{\wedge} 1}$, and define $\eta_{s \wedge 1}$ to be $\nu_{s} \wedge \beta$ for some $\beta$ in $Z_{1}$ larger than $\xi_{s \wedge 0}$. By definition $\xi_{s \wedge 0}$ is in $N_{\eta_{s \wedge 0}}$ and $\xi_{s^{\wedge} 1}$ is in $N_{\eta_{s \wedge 1}}$. 
We claim that if $\eta_{s^{\wedge} 0} \unlhd \nu$ in $T$, then $\xi_{s^{\wedge} 1}$ is not in $N_{\nu}$. Since $\alpha$ is in $Z_{0}, \xi_{s^{\wedge} 1}$ is not in $N_{\left(\eta_{s}{ }^{0}\right)} \cap \alpha$. But $\xi_{s \wedge 1}<\alpha$, so $\xi_{s \wedge 1}$ is not in $N_{\left(\eta_{s^{\wedge} 0}\right)}$. By Lemma 5.6 the minimum element of $N_{\nu} \cap \omega_{2}$ which is not in $N_{\left(\eta_{s} \wedge_{0}\right)}$, if such an ordinal exists, is at least $\sup \left(N_{\left(\eta_{s}{ }_{0}\right)} \cap \omega_{2}\right) \geq \alpha>\xi_{s^{\wedge} 1}$. So $\xi_{s \wedge 1}$ is not in $N_{\nu}$. Similarly if $\eta_{s \wedge 1} \unlhd \nu$ in $T$, then $\xi_{s \wedge 0}$ is not in $N_{\nu}$. This completes the definition. Conditions (1) and (2) are now easily verified.

Since $\mathbb{P}$ is $\omega_{1}$-c.c., the condition $p$ itself is generic for each $N_{\eta}$. Let $G$ be a generic filter for ADD over $V$ which contains $p$. Then for all $\eta$ in $T, N_{\eta}[G] \cap \omega_{2}=N_{\eta} \cap \omega_{2}$. So for any cofinal branch $b$ of $T$ in $V[G], N_{b}[G] \cap \omega_{2}=\bigcup\left\{N_{b\lceil n} \cap \omega_{2}: n<\omega\right\}$; in particular, $N_{b}[G] \cap \omega_{1}=N_{\langle\rangle} \cap \omega_{1}$.

Let $f: \omega \rightarrow 2$ be a function in $V[G] \backslash V$. Define $b_{f}=\bigcup\left\{\eta_{f i n}: n<\omega\right\}$. We prove that $N_{b_{f}} \cap \omega_{2}$ is not in $V$ by showing how to define $f$ inductively from this set. Suppose $f \uparrow n$ is known. Fix $j<2$ such that $f(n) \neq j$. We claim that $\xi^{*}=\xi_{\left(f\lceil n)^{\wedge} j\right.}$ is not in $N_{b_{f}} \cap \omega_{2}$. Otherwise there is $k>n$ such that $\xi^{*}$ is in $N_{\eta_{f \nmid k}}$. But $f \uparrow(n+1) \unlhd f \uparrow k$. So by condition $(2), \xi^{*}$ is not in $N_{\eta_{f \nmid k}}$, which is a contradiction. So $f(n)$ is the unique $i<2$ such that $\xi_{(f\lceil n) \wedge}{ }_{i}$ is in $N_{b_{f}} \cap \omega_{2}$.

Let $\left\langle D_{n}: n<\omega\right\rangle$ enumerate all the dense subsets of $\mathbb{P}(S)$ lying in $N_{b_{f}}[G]$. Inductively define a sequence $\left\langle q_{n}: n<\omega\right\rangle$ by letting $q_{0}=q$ and choosing $q_{n+1}$ to be a refinement of $q$ in $D_{n} \cap N_{b_{f}}[G]$. Let $\left\langle b_{i}: i<\gamma\right\rangle=\bigcup\left\{q_{n}: n<\omega\right\}$. Clearly

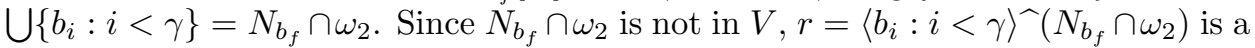
condition in $\mathbb{P}(S)$ below $q$ and $r$ is generic for $N_{b_{f}}[G]$. So $r$ forces $N_{b_{f}}[G][\dot{H}] \cap \omega_{1}=$ $N_{b_{f}}[G] \cap \omega_{1}=N_{\langle\rangle} \cap \omega_{1}$. Let $\dot{r}$ be a name for $r$. Then $p * \dot{r} \leq p * \dot{q}$ is as required.

We state without proof the facts concerning RCS iterations which we shall use. These facts follow immediately from [10] Chapter XI 1.13 and Chapter XV 4.15.

Theorem 5.9. Suppose $\left\langle\mathbb{P}_{i}, \mathbb{Q}_{j}: i \leq \alpha, j<\alpha\right\rangle$ is an $R C S$ iteration. Then $\mathbb{P}_{\alpha}$ preserves $\omega_{1}$ if the iteration satisfies the following properties:

(1) for each $i<\alpha$ there is $n<\omega$ such that $\mathbb{P}_{i+n} \Vdash\left|\mathbb{P}_{i}\right| \leq \omega_{1}$,

(2) for each $i<\alpha$ there is an uncountable regular cardinal $\kappa_{i}$ and a $\mathbb{P}_{i}$-name $\dot{\mathbb{I}}_{i}$ such that $\mathbb{P}_{i}$ is $\kappa_{i}$-c.c. and $\mathbb{P}_{i}$ forces $\dot{\mathbb{I}}_{i}$ is a non-empty restriction-closed $\kappa_{i}$-complete family of ideals such that $\dot{\mathbb{Q}}_{i}$ satisfies the $\dot{\mathbb{I}}_{i}$-universal property.

Theorem 5.10. Let $\alpha$ be a strongly inaccessible cardinal. Suppose that $\left\langle\mathbb{P}_{i}, \mathbb{Q}_{j}\right.$ : $i \leq \alpha, j<\alpha\rangle$ is a revised countable support iteration such that $\mathbb{P}_{\alpha}$ preserves $\omega_{1}$ and for all $i<\alpha,\left|\mathbb{P}_{i}\right|<\alpha$. Then $\mathbb{P}_{\alpha}$ is $\alpha$-c.c.

Suppose $\kappa$ is a Mahlo cardinal and let $A$ be the stationary set of strongly inaccessible cardinals below $\kappa$. Define an RCS iteration $\left\langle\mathbb{P}_{i}, \dot{\mathbb{Q}}_{j}: i \leq \kappa, j<\kappa\right\rangle$ by recursion as follows. Our recursion hypotheses will include that each $\mathbb{P}_{\alpha}$ preserves $\omega_{1}$, and is $\alpha$-c.c. if $\alpha$ is in $A$.

Suppose $\mathbb{P}_{\alpha}$ is defined. If $\alpha$ is not in $A$, then let $\dot{\mathbb{Q}}_{\alpha}$ be a name for $\operatorname{CoLL}\left(\omega_{1},\left|\mathbb{P}_{\alpha}\right|\right)$. Suppose $\alpha$ is in $A$. By the recursion hypotheses $\mathbb{P}_{\alpha}$ forces $\alpha=\omega_{2}$. Let $\dot{\mathbb{Q}}_{\alpha}$ be a name for the poset $\mathrm{ADD} * \mathbb{P}(\dot{S})$.

If $\alpha$ is not in $A$, then choose some regular cardinal $\kappa_{\alpha}$ larger than $\left|\mathbb{P}_{\alpha}\right|$, and let $\dot{\mathbb{I}}_{\alpha}$ be a name for some non-empty restriction-closed $\kappa_{\alpha}$-complete family of ideals on $\kappa_{\alpha}$. Then $\mathbb{P}_{\alpha}$ is $\kappa_{\alpha}$-c.c., and since $\dot{\mathbb{Q}}_{\alpha}$ is proper, $\mathbb{P}_{\alpha}$ forces $\dot{\mathbb{Q}}_{\alpha}$ satisfies the $\dot{\mathbb{I}}_{\alpha^{-}}$ universal property. Suppose $\alpha$ is in $A$. Then let $\alpha=\kappa_{\alpha}$ and define $\dot{\mathbb{I}}_{\alpha}$ as a name for the family of ideals on $\omega_{2}$ as described in Theorem 5.8. Then $\mathbb{P}_{\alpha}$ is $\kappa_{\alpha}$-c.c. and forces that $\dot{\mathbb{Q}}_{\alpha}$ satisfies the $\dot{\mathbb{I}}_{\alpha}$-universal property. 
Suppose $\beta \leq \kappa$ is a limit ordinal and $\mathbb{P}_{\alpha}$ is defined for all $\alpha<\beta$. Define $\mathbb{P}_{\beta}$ as the revised countable support limit of $\left\langle\mathbb{P}_{\alpha}: \alpha<\beta\right\rangle$. By Theorem 5.9 and the recursion hypotheses, $\mathbb{P}_{\beta}$ preserves $\omega_{1}$. Hence if $\beta$ is in $A \cup\{\kappa\}$, then $\mathbb{P}_{\beta}$ is $\beta$-c.c. by Theorem 5.10 .

This completes the definition. Let $G$ be generic for $\mathbb{P}_{\kappa}$. The poset $\mathbb{P}_{\kappa}$ is $\kappa$-c.c. and preserves $\omega_{1}$, so in $V[G]$ we have that $\kappa=\omega_{2}$ and $A$ is a stationary subset of $\omega_{2} \cap \operatorname{cof}\left(\omega_{1}\right)$. For each $\alpha$ in $A$ let $\mathcal{C}_{\alpha}$ be the club on $P_{\omega_{1}}(\alpha)$ introduced by $\mathbb{Q}_{\alpha}$. If $\alpha<\beta$ are in $A$, then $\mathcal{C}_{\alpha}$ and $\mathcal{C}_{\beta}$ are disjoint since $\mathcal{C}_{\beta}$ is disjoint from $V[G \uparrow \beta]$. So $\left\langle\mathcal{C}_{\alpha}: \alpha \in A\right\rangle$ is a disjoint club sequence on $\omega_{2}$ in $V[G]$.

We conclude the paper with several questions.

(1) Assuming Martin's Maximum, the poset $\operatorname{ADD} * \mathbb{P}(\dot{S})$ is semiproper. Is this poset semiproper in general?

(2) Is it consistent that there exists a stationary set $A \subseteq \omega_{2} \cap \operatorname{cof}\left(\omega_{1}\right)$ such that neither $A \cup \operatorname{cof}(\omega)$ nor $\omega_{2} \backslash A$ can acquire a club subset in an $\omega_{1}$ and $\omega_{2}$ preserving extension?

(3) To what extent can the results of this paper be extended to cardinals greater than $\omega_{2}$ ? For example, is it consistent that there is a fat stationary subset of $\omega_{3}$ which cannot acquire a club subset by any forcing poset which preserves $\omega_{1}, \omega_{2}$, and $\omega_{3}$ ?

\section{REFERENCES}

1. U. Abraham and S. Shelah, Forcing closed unbounded sets, Journal of Symbolic Logic 48 (1983), no. 3, 643-657. MR0716625 (85i:03112)

2. J. Baumgartner and A. Taylor, Saturation properties of ideals in generic extensions I, Transactions of the American Mathematical Society 270 (1982), no. 2, 557-574. MR0645330 (83k:03040a)

3. Q. Feng, T. Jech, and J. Zapletal, On the structure of stationary sets, Preprint.

4. M. Foreman, M. Magidor, and S. Shelah, Martin's maximum, saturated ideals, and non-regular ultrafilters. part I, Annals of Mathematics 127 (1988), 1-47. MR0924672 (89f:03043)

5. S. Friedman, Forcing with finite conditions, Preprint.

6. M. Gitik, Nonsplitting subset of $P_{\kappa} \kappa^{+}$, J. Symbolic Logic 50 (1985), no. 4, 881-894. MR 0820120 (87g:03054)

7. W. Mitchell, $I\left[\omega_{2}\right]$ can be the non-stationary ideal, Preprint.

8. - Aronszajn trees and the independence of the transfer property, Annals of Mathematical Logic 5 (1972), 21-46. MR0313057 (47:1612)

9. M. Rubin and S. Shelah, Combinatorial problems on trees: Partitions, $\Delta$-systems and large free subtrees, Annals of Pure and Applied Logic 33 (1987), 43-81. MR0870686 (88h:04005)

10. S. Shelah, Proper and improper forcing, Springer, 1998. MR1623206 (98m:03002)

Kurt Gödel Research Center for Mathematical Logic, University of Vienna, Waehringer Strasse 25, A-1090 Wien, Austria

E-mail address: sdf@logic.univie.ac.at

Department of Mathematics, University of California, Berkeley, California 94720

E-mail address: jkrueger@math.berkeley.edu 